\title{
ELABORACIÓN DE UN TRABAJO PRÁCTICO SOBRE LA ANTROPOLOGÍA DE LOS CUIDADOS
}

* Miralles Sangro, Ma Teresa. ** Casas Martínez, Ma Francisca. *** González Villanueva, Purificación

* Enfermera Hospital Ramón y Cajál. Profesora Asc. EUE y F Univ. de Alcalá

** Profesora Titular Int. De Enfermería Materno Infantil. EUE y F Unv. de Alcalá

*** Enfermera Hospital Ramón y Cajál. Profesora Asc. EUE y F Univ. de Alcalá

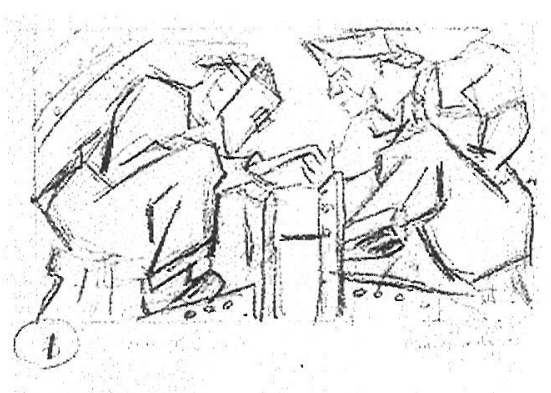

«Soldadores» de Chaim Gross Welders I (sketch for relief panel) 1937 , U. S. Federal Trade Commission

Correspondencia: $\mathrm{M}^{\mathrm{a}}$ Teresa Miralles Sangro

Samaria $n^{\circ} 4,3^{\circ}$ izq. 28009 Madrid

Teléfono $91 / 409.38 .98$

\section{RESUMEN}

Este artículo presenta la metodología propuesta a los alumnos para realizar el Trabajo Práctico obligatorio, en la Asignatura de Antropología del Cuidado en la Escuela Universitaria de Enfermería de Alcalá. La finalidad de este trabajo consta de dos apartados:

1.- Que el alumno conozca los aspectos del cuidado como experiencia humana en la diversidad cultural.

2.- Las características y estructura para su realización y presentación.

Los resultados muestran que la realización de un Trabajo Práctico por los alumnos en la Asignatura de Antropología del Cuidado les aporta una visión global y comparativa de los cuidados cotidianos, permitiéndoles buscar unas formas de ayuda más eficaces y adecuadas, al considerar el cuidado como una experiencia humana y universal.

El Trabajo Práctico aumenta el interés del alumno por la Asignatura y mejora el nivel de conocimientos sobre los cuidados.

Palabras clave: Investigación. Cuidados. Antropología. Cuidados cotidianos.

\section{PRACTICAL WORK ABOUT ANTHROPOLOGY OF NURSING CARE}

\begin{abstract}
The present article shows a proprosal of methodological work given to students with the aim of preparing their compulsory Practical Work on the Subject: «Anthropology of the Nursing Care», at the Nursing School in the University of Alcalá

The aim of this study is divided in two parts:

1. The student will know the aspects of «nursing care» as a human experience in the different cultures.

2. The characteristics and structures for its implementation and presentation.

The results show that a Practical Work prepared by the students of the Subject: «Anthropology of the Nursing Care», furnish them with a global and comparative vision of the day-to-day nursing care, encouraging in them the search of new, more efficient and more adequate ways of helping patients/clients to consider nursing care as a human and universal experience.
\end{abstract}

Key words: Research. Nursng care. Anthropology. Day-to-day nursing care. 


\section{INTRODUCCIÓN}

En la Escuela Universitaria de Enfermería y Fisioterapia de Alcalá la asignatura Antropología del Cuidado se contempla dentro del curriculum básico como materia optativa. El objetivo de esta asignatura es aumentar los conocimientos sobre la diversidad cultural y contribuir a la sensibilización y comprensión tanto de otras culturas, como de la propia, con el fin de alcanzar la máxima eficacia en el campo de los cuidados. La metodología didáctica utilizada comprende, además de las clases teóricas, la realización obligatoria de un Trabajo Práctico, en grupos de 465 alumnos, utilizando las herramientas que le proporciona la antropología.

El objetivo de este Trabajo Práctico es que el alumno tome conciencia y/o viva situaciones que le permitan reflexionar sobre los cuidados como una de las experiencias humanas, que le han permitido al hombre sobrevivir y expandirse hasta configurarse como las personas que somos actualmente (Collière, 1993). Se pretende que el alumno reconozca la diversidad cultural, los distintos modos de percibir la buena y la mala salud y los medios que utilizan los diferentes grupos sociales para proporcionar cuidados. Desarrolle estrategias de investigación y proponga alternativas o iniciativas, desde la visión enfermera, para el mantenimiento, promoción y protección de la salud de las personas (Collière, 1994).

\section{DESARROLLO}

Para la elaboración del trabajo se ofrece a los alumnos un listado de títulos que recogen aspectos sobre el origen de los cuidados, la evolución del hombre, la familia y la organización doméstica, la cultura, el lenguaje, la

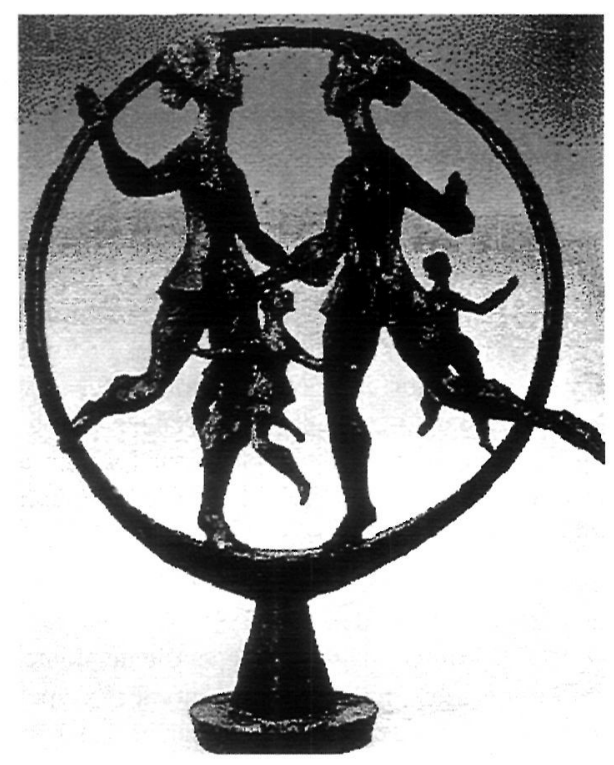

marginalidad, la organización de los cuidados en función del rol, la religión, los ritos en las etapas de la vida

Los principales pasos en la realización del Trabajo comprenden:

Recopilación de la información; bibliografía, lecturas recomendadas, artículos de actualidad (tanto profesionales como sociales; prensa, revistas), iconografía, todos ellos indispensables para conocer los estudios y la información existente relacionada con el tema. El alumno debe inspirarse en los últimos resultados de los estudios científicos, en sus principios y en sus objetivos.

Tener una buena preparación teórica y estar al tanto de los datos más recientes no es lo mismo que esta cargado de «ideas preconcebidas». Cuantos más problemas se plantee el alumno sobre la marcha, cuanto más se acostumbre a moldear sus teorías a los hechos y a ver los datos como capaces de configurar una teoría, mejor equipado estará para su trabajo. Las ideas preconcebidas son perniciosas en todo trabajo científico, pero las conjeturas son el don principal de un pensador científico, y tales conjeturas le son posibles al observador sólo gracias a sus estudios teóricos.

Cuando el alumno decide recoger los datos de un Centro concreto, es indispensable que redacte una Carta de Presentación, para solicitar la información, como alumnos de enfermería interesados en aspectos relacionados con el Trabajo que allí se desarrolla y dirigida a la persona adecuada de las Instituciones, Organismos o Centros donde se realizará la investigación o la visita.

Para recolectar los datos el alumno puede utilizar diferentes técnicas (Kottak, 1994):

- Técnicas de observación. Observación directa, que le permite prestar atención y recoger cientos de detalles de la vida cotidiana, registrando aquello que ve tal como lo ve (destrezas, actitudes, actividades, conductas, utensilios). Es importante registrar las impresiones lo antes posible en el curso del trabajo, pues ciertas peculiaridades sutiles sólo llaman la atención mientras son nuevas, dejando de percibirse tan pronto como se hacen familiares. Observación participante, tomando parte en la vida de la comunidad al tiempo que se estudia, facilita la comprensión de los hechos o procesos que estamos estudiando; por ejemplo si estamos investigando las funciones que cumplen las ONG, sería bueno entablar relación y participar en alguna de sus actividades.

- Conversaciones más o menos formales que contribuyen a mantener la relación y permiten ponerse al día «de lo que pasa». Es necesario comprender la oratoria especial de cada grupo; liturgias de ceremonias políticas y rituales religiosos, ritos de higiene, lenguaje del cuidado. 
- Entrevista pautada, con cuestionario, para asegurarnos una información completa y comparable de los aspectos de interés del estudio. En este caso el investigador habla cara a cara con sus informantes, pero los procedimientos del cuestionario tienden a ser más indirectos e impersonales.

- El método genealógico, descubriendo y registrando vínculos de parentesco, filiación y matrimonio, bases de la organización social. En la investigación del parentesco, siguiendo una tras otra todas las relaciones de un caso concreto, se desemboca de forma natural en la construcción de cuadros genealógicos. Los datos genealógicos valdrán para reconstruir la historia y entender las relaciones actuales dentro del grupo y para conocer los roles que determina la comunidad a cada individuo. Se utiliza la representación visual del parentesco para situar a las personas, por lo que resulta necesario conocer la simbología de los signos y códigos utilizados en la antropología. A la hora de planificar cuidados es interesante conocer los vínculos del parentesco de la persona que requiere los cuidados, para saber con quién se cuenta, como familia, para mantener los cuidados.

- Historias de vida; nos permitirán conocer como perciben, reaccionan y contribuyen a cambios que afectan a sus vidas, determinadas personas concretas del grupo. Esta recogida de las experiencias de toda una vida proporciona un retrato cultural, más personal, íntimo y difícil de obtener por otros medios. La organización de los cuidados en función del rol que una determinada sociedad atribuye a cada individuo la podremos conocer a través de las Historias de vida.

- Investigación centrada sobre temas o problemas concretos.

- Trabajo de campo, objeto y aspiraciones; el objetivo del trabajo etnográfico del campo es dar un esquema claro y coherente de la estructura social y destacar, de entre el cúmulo de hechos irrelevantes, las leyes y normas que todo fenómeno cultural conlleva.

La meta del trabajo de campo debe alcanzarse a través de tres vías:

1) La organización del grupo y la anatomía de su cultura que deben recogerse en un esquema preciso y claro. Para lo cual el alumno, debe colocarse en condiciones adecuadas para su trabajo; a través de un trato natural con las personas del grupo estudiado, dejándole que cuente anécdotas, discutiendo sus costumbres, familiarizándose con sus creencias, los detalles íntimos de la vida familiar, el aseo, la cocina, las peleas, las bromas, los ritos. El sa- ber gozar de la observación, el aceptar a la persona como primera fuente de conocimientos es la condición previa para llevar a cabo con éxito cualquier trabajo de campo. El método de documentación concreta estadística es el medio que permite construir tal esquema.

2) Dentro de este entramado hay que insertar los imponderables de la vida real y el tipo de comportamiento. Estos datos se consiguen gracias a la observación minuciosa y detallada, en forma de una especie de diario etnográfico, posible a partir de un estrecho contacto con la vida del grupo.

3) Los informes, narraciones características, expresiones típicas, datos del folklore y fórmulas mágicas se agruparán como datos representativos de la mentalidad del grupo.

El trabajo de campo es también un rito de paso. Se dice de la experiencia de campo que es radicalmente auto-transformadora. Una vez recogidos los datos se vierte la información (todos los aspectos de la vida de la comunidad), en la medida de lo posible, en gráficos o cuadros sinópticos para a continuación poder identificar, analizar y comparar con su referente, para poder explicar las diferencias, similitudes y particularidades del grupo estudiado. Cualquier tipo de transacción económica puede estudiarse por el procedimiento de seguir casos reales conectados y traspasarlos luego a un cuadro sinóptico. Del mismo modo, podemos trazar un cuadro de todas las ofrendas y regalos habituales en una sociedad dada, incluyendo la definición sociológica, ceremonial y económica de cada artículo. De la misma forma se pueden recoger en un cuadro todos los cuidados cotidianos y habituales del grupo, cuidados alrededor del cuerpo, cuidados sobre la alimentación, cuidados en relación con salud, términos que se utilizan para los cuidados. También los sistemas de magia, las series de ceremonias interrelacionadas, los ritos de paso, los diversos tipos de actos legales. Todo puede ser fichado de modo que cada elemento pueda determinarse sinópticamente bajo cierto número de epígrafes (Harris, 1994).

En los resultados de la investigación constarán todas las condiciones del estudio: manera en que fueron encauzadas las observaciones, su número y el tiempo dedicado. También se hará distinción entre, por una parte, lo que son los resultados de la observación directa, exposiciones e interpretaciones de las personas del grupo estudiado y, por otra parte, las deducciones de los autores basadas en su sentido común y capacidad de comprensión (Polit \& Hungler, 1996).

En cuanto a la concepción y diseño, el trabajo se debe ajustar a las normas de Vancouver. (Icart Isern y Caja López, 1995): Primera página, independiente, conteniendo el título, resumen, palabras clave, nombre y apellidos de los autores, lugar de trabajo. Desarrollo del trabajo 
propiamente: Introducción, material y método, resultados, discusión, agradecimientos, bibliografía, figuras.

La presentación de los Trabajos de investigación se realiza en una sesión en la que participan la totalidad de los alumnos, contando cada grupo con un tiempo de exposición de veinte minutos en el que pueden utilizar cualquier soporte visual.

\section{CONCLUSIÓN}

En nuestra valoración podemos constatar que:

- El Trabajo Práctico sobre la Antropología de los Cuidados aumenta el interés de los alumnos por la asignatura. dos.

- Mejora el nivel de conocimientos sobre los cuida-

- Estimula la observación del alumno y le impulsa a considerar a la persona cuidada como primera fuente de conocimientos. Permitiéndole buscar unas formas de ayuda $m$ 'ss eficaces y adecuadas al considerar al cuidado como una experiencia humana y universal.

\section{BIBLIOGRAFÍA}

Collière, M. F. (1993). Promover la vida. Interamericana, Madrid.

Collière, M. F. (1994). Soigner, Le premier art de la vie. Inter Editions, París.

Kottak, C. P. (1994). Antropología. Una exploración de la diversidad humana. McGraw Hill, Madrid.

Harris, M. (1994). Introducción a la Antropología General. Alianza Universidad Textos, Madrid.

Polit, D. F. y Hungler, B, P. (1996). Investigación científica en ciencias de la salud. Interamericana -McGraw Hill, Madrid.

Icart Isern, T. y Caja López, C. (1995). Cómo escribir un artículo «original» para una revista científica. Enfermería Clínica 4/ 2, 36-40.

\section{MATERIAL}

- Sellos de Correos pertenecientes a la colección particular de $D^{a}$. M $M^{\text {a }}$ Miralles Sangro 